\title{
Improving chromosome synthesis with a semi-quantitative phenotypic assay and refined assembly strategy
}

\section{Supplementary Material}

Yicong Lin ${ }^{1,2}$, Xinzhi Zou ${ }^{1, \#}$, Yihui Zheng ${ }^{1}$, Yizhi Cai ${ }^{2,3, *}$, Junbiao Dai ${ }^{1,2, *}$

${ }^{1}$ Key Laboratory of Industrial Biocatalysis (Ministry of Education) and Center for Synthetic and Systems Biology, School of Life Sciences, Tsinghua University, Beijing 100084, China

${ }^{2}$ Shenzhen Key Laboratory of Synthetic Genomics and Center for Synthetic Genomics, Institute of Synthetic Biology, Shenzhen Institutes of Advanced Technology, Chinese Academy of Sciences, Shenzhen 518055, China

${ }^{3}$ Manchester Institute of Biotechnology, University of Manchester, 131 Princess Street, M1 7DN, Manchester, UK

\# Current address: Department of Bioengineering, Stanford University, Stanford, California, USA 
Table S1. sgRNA sequences

\begin{tabular}{|l|l|}
\hline Name & Sequence \\
\hline sgRNA-1 & AATCACAGCCGAAGCCATTA \\
\hline sgRNA-2 & ACCCACAGCACCTAACAAAA \\
\hline
\end{tabular}




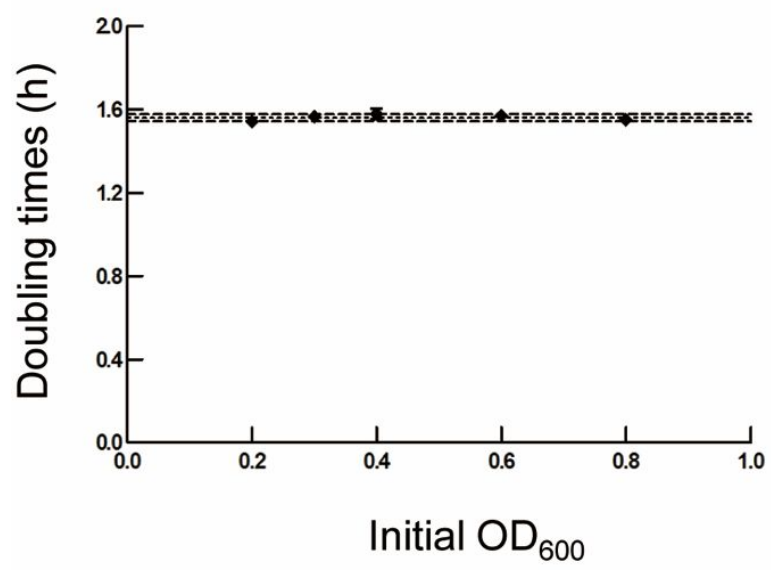

Figure S1. Scatter plot showing doubling times of cultures with different initial cell densities. Mean doubling time is shown by a dotted line, and $95 \%$ confidence intervals are showed by dashed lines. 

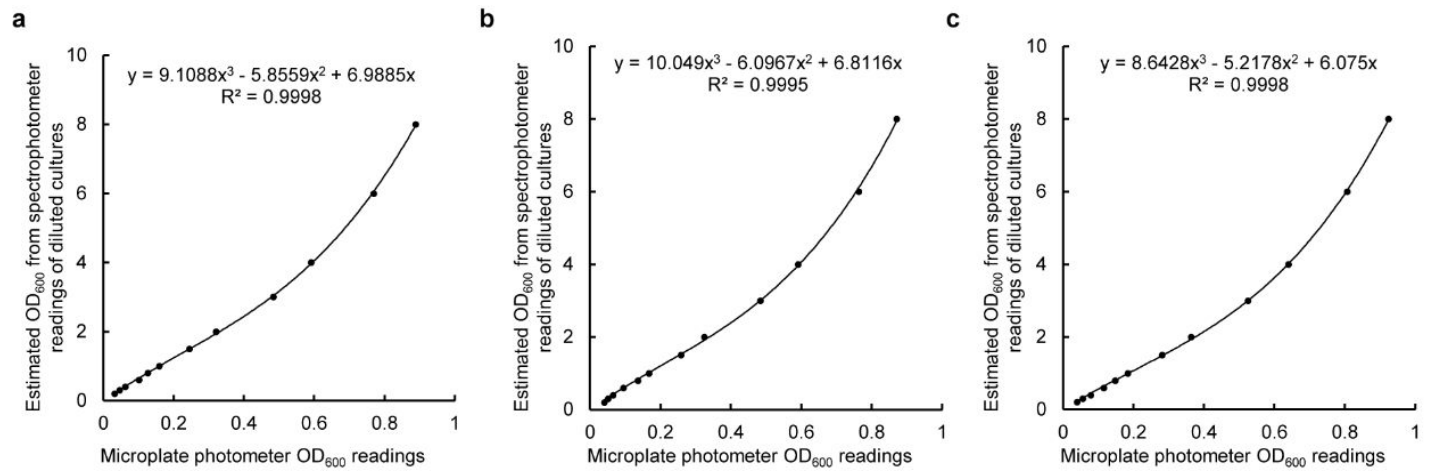

Figure S2. Calibration curves of estimated $\mathrm{OD}_{600}$ from spectrophotometer readings of diluted cultures vs. microplate photometer readings in YPD (a), YPD $+6 \mathrm{mM} \mathrm{CuSO}_{4}$ (b), and YPD + 1M NaCl media (c). 


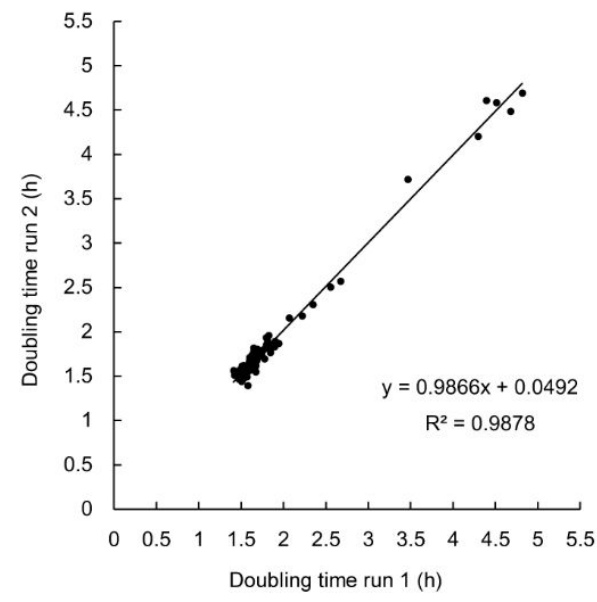

Figure S3. Scatter plot of doubling times the five knockout strains under all test conditions in two experimental runs. $\mathrm{R}^{2}$ and the slope of the regression line were close to 1 , indicating that our assay was highly reproducible. 


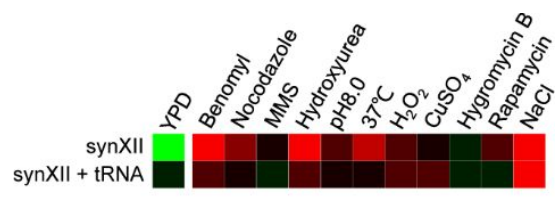

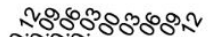

$0,0,0,0.000 .0$.

Figure S4. Heat map showing the LSC $_{\mathrm{YPD}}$ and LPI of synXII strains under all eleven stress conditions. 


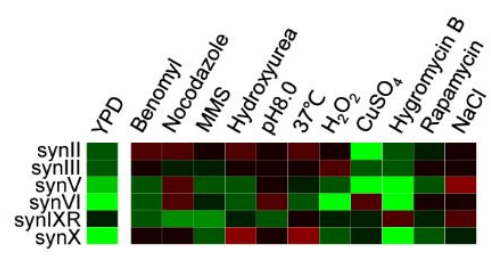

520.303025

0.0 .000 .0001

b

\begin{tabular}{|c|c|c|c|}
\hline \multirow{2}{*}{\multicolumn{2}{|c|}{ BY4741 }} & YPD, 36h & YPD, 48h \\
\hline & & 00 再 & 20 \\
\hline & 00 䇣 & 00 箦 & 00 क \\
\hline & 00 & 00 & 00 缽 \\
\hline & 00 & 00 竫 & $00 \div$ \\
\hline
\end{tabular}

Figure S5. (a) Heat map showing $\mathrm{LSC}_{\mathrm{YPD}}$ and LPI under all stress conditions of synII, synIII, synV, synVI and synX. (b) Spotting assay results showing the growth of synV, synVI, and synX on YPD plates. 


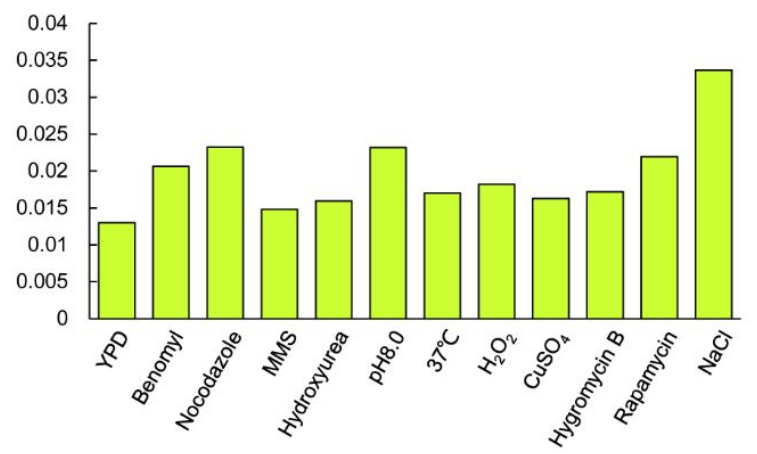

Figure S6. Mean standard deviations under each test condition. 